\title{
Multi-hop Reading Comprehension across Documents with Path-based Graph Convolutional Network
}

\author{
Zeyun Tang, Yongliang Shen, Xinyin Ma, Wei Xu, Jiale Yu and Weiming $\mathrm{Lu}^{*}$ \\ College of Computer Science and Technology, Zhejiang University, China \\ luwm@zju.edu.cn
}

\begin{abstract}
Multi-hop reading comprehension across multiple documents attracts much attention recently. In this paper, we propose a novel approach to tackle this multi-hop reading comprehension problem. Inspired by human reasoning processing, we construct a path-based reasoning graph from supporting documents. This graph can combine both the idea of the graph-based and path-based approaches, so it is better for multi-hop reasoning. Meanwhile, we propose Gated-RGCN to accumulate evidence on the path-based reasoning graph, which contains a new question-aware gating mechanism to regulate the usefulness of information propagating across documents and add question information during reasoning. We evaluate our approach on WikiHop dataset, and our approach achieves state-of-the-art accuracy against previously published approaches. Especially, our ensemble model surpasses human performance by $4.2 \%$.
\end{abstract}

\section{Introduction}

Machine reading comprehension has been a popular topic in the past years, and a variety of models have been proposed to address this problem, such as BIDAF [Seo et al., 2017], Reinforced mnemonic reader [Hu et al., 2017], and ReasoNet [Shen et al., 2017]. However, most existing works focus on finding evidence and answer in a single document.

In fact, in reality, many questions can only be answered after reasoning across multiple documents. Table 1 shows a multi-choice style reading comprehension example, which is from WIKIHOP dataset [Welbl et al., 2018]. In the example, we can only answer the question "what is the place of death of alexander john ellis?' after extracting and integrating the facts 'Alexander John Ellis is buried in Kensal Green Cemetery' and 'Kensal Green Cemetery is located in Kensington' from multiple documents, which is a more challenging task.

The main challenge is that the evidence is distributed in different documents and there is a lot of noise in the documents. We need to extract this evidence from multiple documents, but it is difficult to capture their dependencies for reasoning.

\footnotetext{
${ }^{*}$ corresponding author
}

\begin{tabular}{l}
\hline Question: place of death, alexander john ellis, ? \\
\hline Support doc1: Alexander John Ellis, was an English \\
mathematician ... is buried in Kensal Green Cemetery. \\
Support doc2: The areas of College Park and Kensal \\
Green Cemetery are located in the London boroughs of \\
Hammersmith \& Fulham and Kensington \& Chelsea, re- \\
spectively. \\
..... \\
\hline Candidates: college park, france, Kensington, London \\
\hline Answer: Kensington \\
\hline
\end{tabular}

Table 1: An example of multi-hop reading comprehension across documents.

Many works used graph convolution networks(GCNs) to deal with this problem, such as Entity-GCN [De Cao et al., 2019], BAG [Cao et al., 2019] and HDE [Tu et al., 2019]. They transform documents into an entity graph, and then import the entity graph into graph convolution networks(GCNs) to simulate the process of multi-hop reasoning.

However, these GCN-based approaches have some disadvantages. Firstly, they generated the entities only from the question and candidate answers, lacking much key information for multi-hop reasoning. For example, as the example in Table 1, the entity 'Kensal Green Cemetery' is an important clue to answer the question, but the above approaches ignored this information. Secondly, the traditional GCNs only update the central node based on the aggregated information of adjacent nodes and use this to simulate the process of reasoning. But the question information is not fully utilized and there is a lot of irrelevant information during information propagating across documents in the multi-hop reasoning.

In this paper, we propose a novel approach to solve the above problem. We introduce a path-based reasoning graph for multiple documents. Compared to traditional graphs, the path-based reasoning graph contains multiple reasoning paths from questions to candidate answers, combining both the idea of the GCN-based and path-based approaches. Thus, we construct a path-based reasoning graph by extracting reasoning paths(e.g., Alexander John Ellis $\rightarrow$ Kensal Green Cemetery $\rightarrow$ Kensington) from supporting documents and then adding reasoning nodes (e.g., Kensal Green Cemetery) in these path- 
s to the entity graph. And then, we apply a Gated-RGCN to learn the representation of nodes. Compared to GCNs, GatedRGCN utilizes attention and question-aware gating mechanism to regulate the usefulness of information propagating across documents and add question information during reasoning, which is closer to human reasoning processes.

Our contributions can be summarized as follows:

- We propose a path-based reasoning graph, which introduces information about reasoning paths into the graph;

- We propose Gated-RGCN to optimize the convolution formula of RGCN, which is more suitable for multi-hop reading comprehension;

- We evaluated our approach on WIKIHOP dataset [Welbl et al., 2018], and our approach achieves new state-ofthe-art accuracy. Especially, our ensemble model surpasses the human performance by $4.2 \%$.

\section{Related Work}

Recently, there are several categories of approaches that have been proposed to tackle the problem of multi-hop reading comprehension across documents, including GCN-based approaches (Entity-GCN [De Cao et al., 2019], BAG [Cao et al., 2019], HDE [Tu et al., 2019], MHQA-GRN [Song et al., 2018], DFGN [Qiu et al., 2019]), memory based approaches (Coref-GRU [Dhingra et al., 2018], EPAr [Jiang et al., 2019]), path based approaches (PathNet [Kundu et al., 2019]), and attention based approaches (CFC [Zhong et al., 2019], DynSAN [Zhuang and Wang, 2019]).

GCN-based approaches organize supporting documents into a graph, and then employ Graph Neural Networks based message passing algorithms to perform multi-step reasoning. For example, Entity-GCN [De Cao et al., 2019] constructed an entity graph from supporting documents, where nodes are mentions of subject entity and candidates, and edges are relations between mentions. BAG [Cao et al., 2019] applied bi-directional attention between the entity graph and the query after GCN reasoning over the entity graph. HDE [Tu et al., 2019] constructed a heterogeneous graph where nodes correspond to candidates, documents, and entities. MHQAGRN [Song et al., 2018] constructed a graph where each node is either an entity mention or a pronoun representing an entity, and edges fall into three types: same-typed, window-typed and coreference-typed edge. DFGN [Qiu et al., 2019] proposed a dynamic fusion reasoning block based on graph neural networks. Our work proposes Gated-RGCN to optimize the graph convolution operation, it is better for regulating the usefulness of information propagating across documents and add question information during reasoning.

Memory-based approaches try to aggregate evidences for each entity from multiple documents through a memory network. For example, Coref-GRU [Dhingra et al., 2018] aggregated information from multiple mentions of the same entity by incorporating coreference in the GRU layers. EPAr [Jiang et al., 2019] used a hierarchical memory network to construct a 'reasoning tree', which contains a set of root-to-leaf reasoning chains, and then merged evidences from all chains to make the final prediction.
PathNet [Kundu et al., 2019] proposed a typical path-based approach for multi-hop reading comprehension. It extracted paths from documents for each candidate given a question, and then predicted the answer by scoring these paths. Our work introduces the idea of path-based approach on GCNbased approach which is better for multi-hop reasoning.

CFC [Zhong et al., 2019] and DynSAN [Zhuang and Wang, 2019] are two typical attention-based approaches. CFC applied co-attention and self-attention to learn query aware node representations of candidates, documents and entities. While DynSAN proposed a dynamic self-attention architecture to determine what tokens are important for constructing intra-passage or cross-passage token level semantic representations. In our work, we employ an attention mechanism between graphs and the question at each layer of GatedRGCN.

Meanwhile, in order to promote the research on multihop QA, several datasets have been designed, including WikiHop [Welbl et al., 2018], OpenBookQA [Mihaylov et al., 2018], NarrativeQA [Kociský et al., 2017], MultiRC [Khashabi et al., 2018] and HotpotQA [Yang et al., 2018]. For example, WikiHop is a multi-choice style reading comprehension data set, where the task is to select the correct object entity from candidates when given a query $\langle s, r, ?\rangle$ and a set of supporting documents. While OpenBookQA focuses on the multi-hop QA which needs a corpus of provided science facts (open book) with external broad common knowledge.

In addition, knowledge completion over knowledge graph $(K G)$ and $K G$ based query answering are also related to our task, since they both need multi-hop reasoning, i.e., finding the reasoning path between two entities in KG. For example, MINERVA [Das et al., 2017] formulates the multi-hop reasoning as a sequential decision problem, and uses the REINFORCE algorithm [Williams, 1992] to train an end-to-end model for multi-hop KG query answering. Meta-KGR [Lv et al., 2019] also used the reinforcement learning method to learn a relation-specific multi-hop reasoning agent to search for reasoning paths and target entities. They further used meta-learning to perform multi-hop reasoning over few-shot relations of knowledge graphs.

\section{Approach}

In this section, we first formulate the task of multi-hop reading comprehension across documents, and then elaborate our approach in detail.

\subsection{Task Formulation}

The task of multi-hop reading comprehension across documents can be formally defined as: given a question $q=$ $\left(q_{1}, q_{2}, \ldots, q_{M}\right)$ and a set of supporting documents $S_{q}$, the task is to find the correct answer $a$ from a set of answer candidates $C_{q}=\left(c_{1}, c_{2}, \ldots, c_{N}\right)$, where $M$ is the number of words in the question $q$ and $N$ is the number of candidates in $C_{q}$.

In the WIKIHOP dataset [Welbl et al., 2018], the question $q$ is given in the form of a tuple $\langle s, r, ?\rangle$, where $s$ represents the subject entity, and $r$ represents the relation between $s$ and the unknown tail entity. In our example, $q=$ 


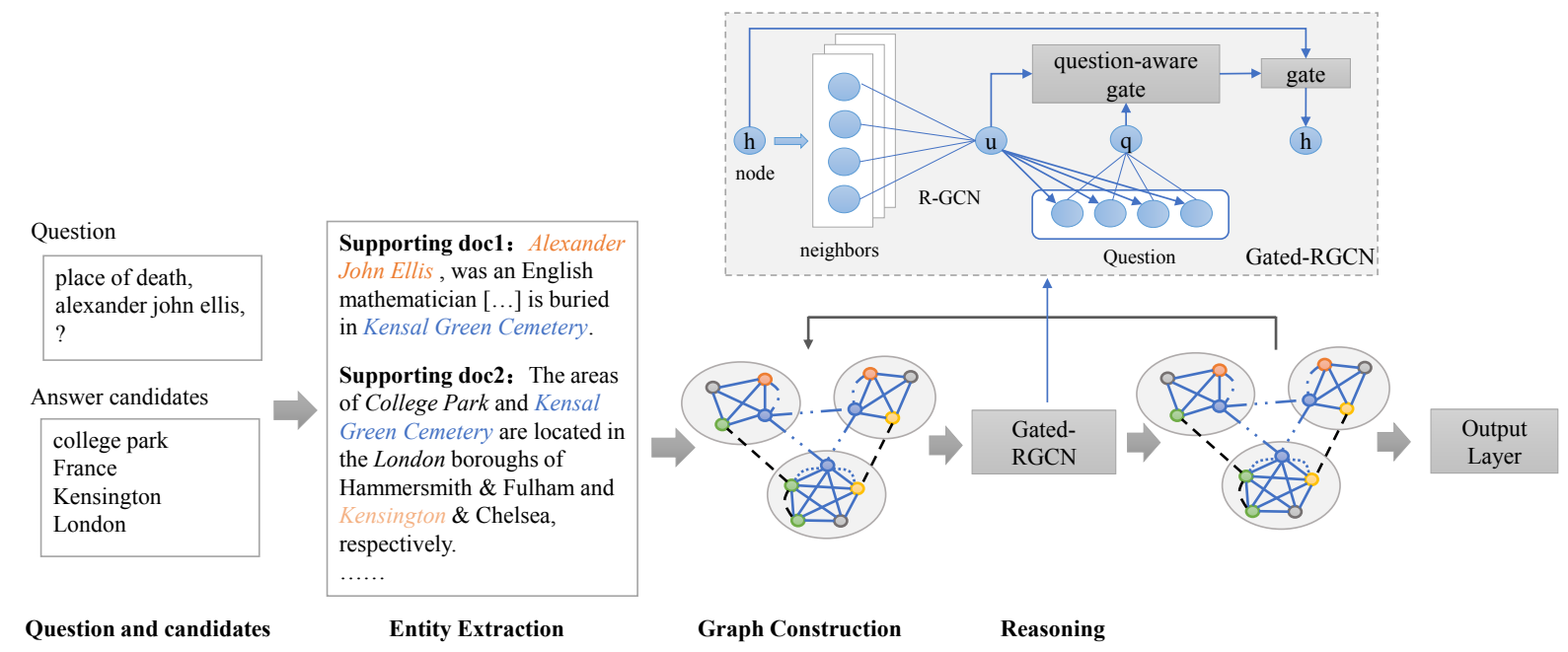

Figure 1: Overview of our approach

$\langle$ alexander john ellis, place of death, ?〉 means where did alexander john ellis die, and the answer candidates is $C_{q}=$ (college park, france, Kensington, London). When given the supporting documents, e.g., supporting docl, doc2 in Table 1, we should identify the correct answer $a=$ Kensington from the candidates by reasoning across these documents.

As shown in Figure 1, our approach mainly consists of three components, including graph construction, reasoning with Gated-RGCN, and output layer. In the following sections, we will elaborate on each component in detail.

\subsection{Graph Construction}

We construct an entity graph based on the EntityGCN [De Cao et al., 2019], which extracts all mentions of entities in $C_{q} \cup\{s\}$ in $S_{q}$ as nodes in the graph. Besides, inspired by the human reasoning processing, reasoning paths from the subject entity in question to the candidates could be helpful for reasoning across documents, so we add reasoning entities in the paths into our entity graph. In our example, the path alexander john ellis $\rightarrow$ Kensal Green Cemetery $\rightarrow$ Kensington from documents indicate that the candidate Kensington may be the correct answer for the question 〈alexander john ellis, place of death, ? $\rangle$. Thus, we treat Kensal Green Cemetery as a reasoning entity, and add it into the entity graph.

Formally, for a given question $q=\langle s, r, ?\rangle$, we would like to extract paths from $s$ to $c_{i} \in C_{q}$ from $S_{q}$, e.g., $p_{i}=s \rightarrow e_{1} \rightarrow e_{2} \rightarrow \ldots \rightarrow e_{l} \rightarrow c_{i}$, where $e_{i}$ is a reasoning entity. In order to find a path, we first find a document $d_{1}$ which contains the mention $m_{s}$ of the subject entity $s$ in $S_{q}$, and then find all the named entities and noun phrases that appear in the same sentence with $m_{s}$. In our example, we find Kensal Green Cemetery and alexander john ellis appear in the same sentence in supporting docl, so we extract Kensal Green Cemetery as one of reasoning entities. Then, we find another document $d_{2}$ which contains any of the reasoning entities. In our example, supporting doc 2 contains the reasoning entity Kensal Green Cemetery. Finally, we check whether the reasoning entity appears with one of the candidates in the same sentence. If so, we would add the path to the entity graph. For example, Kensal Green Cemetery and Kensington appear in the same sentence in supporting doc2. Therefore, the path alexander john ellis $\rightarrow$ Kensal Green Cemetery $\rightarrow$ Kensington can be added to the entity graph.

Since each entity in different documents has different contexts, so we use mentions of the subject entity, reasoning entities, and candidate answers as nodes in the entity graph. In our example, Kensal Green Cemetery appears in two different sentences, so we need to add nodes for different positions Figure 2 shows an example of an entity graph, where $m_{s}, m_{c}$ and $m_{a}$ are mentions of the subject entity $s$, reasoning entities $C_{c}$, and candidate answers $C_{q}$ respectively.

Then, we define the following types of edges between pairs of nodes to encode various structural information in the entity graph.

1. an edge between a subject node and a reasoning node if they appear in the same sentence in a document, e.g., $e_{s c}$ in Figure 2.

2. an edge between two nodes if they are reasoning nodes and are adjacent nodes on the same path, e.g., $e_{c c}$ in Figure 2 .

3. an edge between a reasoning node and a candidate node if they appear in the same sentence in a document, e.g., $e_{c a}$ in Figure 2.

4. an edge between two nodes if they are mentions of the same candidate, e.g., $e_{a a}$ in Figure 2.

5. an edge between two nodes if they appear in the same document.

6. nodes that do not meet previous conditions are connected.

\subsection{Reasoning with Gated-RGCN}

We first use pretrained word embeddings GLoVe [Pennington et al., 2014] to represent each node in the entity graph, and 


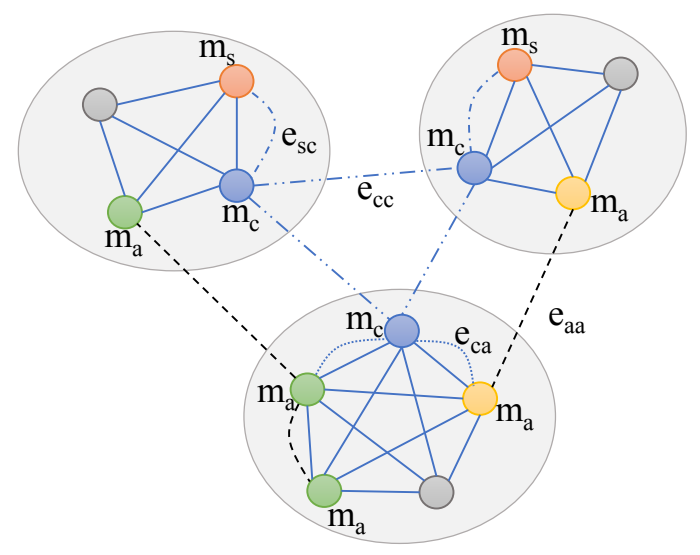

Figure 2: The entity graph for supporting documents (ellipses), where nodes are mentions of the subject entity $\left(m_{s}\right)$, reasoning entities $\left(m_{c}\right)$, and candidates $\left(m_{a}\right)$. Nodes with same color indicate they refer to the same entity. Meanwhile, nodes are connected by six types of edges, which are elaborated in Section 3.2.

then use ELMo [Peters et al., 2018] to model contextual information for each node in different documents. These two vectors are concatenated, and then encoded through 1-layer linear network. Thus, the features for all nodes can be denoted as $f_{n} \in \mathbb{R}^{T \times d}$, where $T$ is the number of nodes in the graph, and $d$ is the dimension of the node feature.

After graph initialization, we employ a Gated Relational Graph Convolutional Network (Gated-RGCN) to realize multi-hop reasoning. First, we use R-GCN to aggregate messages from its direct neighbors. Specifically, at lth layer, the aggregated message $z_{i}^{l}$ for node $i$ can be obtained via

$$
z_{i}^{l}=\sum_{j \in \mathcal{N}_{i}} \sum_{r \in R_{i j}} \frac{1}{\left|\mathcal{N}_{i}\right|} W_{r}^{l} h_{j}^{l}
$$

where $\mathcal{N}_{i}$ is the neighbors of node $i, R_{i j}$ is the set of relations between $i$ and $j, W_{r}^{l} \in \mathbb{R}^{d \times d}$ is a relation-specific weight matrix, $|\cdot|$ indicates the size of $\mathcal{N}_{i}$, and $h_{j}^{l}$ is the hidden state of node $j$ at $l$ th layer. Then, the update message $u_{i}^{l}$ for node $i$ can be obtained by combining the aggregated message with its original node information:

$$
u_{i}^{l}=W_{0}^{l} h_{i}^{l}+z_{i}^{l}
$$

where $W_{0}^{l} \in \mathbb{R}^{d \times d}$ is a general weight.

In Entity-GCN [De Cao et al., 2019], HDE [Tu et al., 2019] and BAG [Cao et al., 2019], a gating mechanism is applied on the update vector $u_{i}^{l}$ and the hidden state of node $i$ for updating the hidden state of node $i$ at the next layer.

$$
\begin{aligned}
w_{i}^{l} & =\sigma\left(f_{g}\left(\left[u_{i}^{l} ; h_{i}^{l}\right]\right)\right) \\
h_{i}^{l+1} & =w_{i}^{l} \odot \tanh \left(u_{i}^{l}\right)+\left(1-w_{i}^{l}\right) \odot h_{i}^{l}
\end{aligned}
$$

where $\sigma$ is the sigmoid function, $\left[u_{i}^{l} ; h_{i}^{l}\right]$ is the concatenation of $u_{i}^{l}$ and $h_{i}^{l}, f_{g}$ is implemented with a single-layer multilayer perceptron (MLP), $\tanh (\cdot)$ is a non-linear activation function, and $\odot$ denotes element-wise multiplication.

This gating mechanism regulates how much of the update message propagates to the next step, so it can prevent overwriting past information. However, the traditional GCN method only updates the central node based on the aggregated information of adjacent nodes, but there is a lot of irrelevant information during information propagating.

When humans do reasoning problems, they always choose the supports information based on the query information. Inspired by human reasoning processing, we add another question-aware gate to optimize graph convolution procedure, which is suitable for multi-hop reading comprehension. This gating mechanism can regulate the aggregated message according to the question, and introduce the question information into the update message simultaneously.

First, we represent the question $q$ using a bidirectional LSTM (BiLSTM) network [Hochreiter and Schmidhuber, 1997], where GLoVe is used as word embeddings.

$$
p=\operatorname{BiLSTM}(q), p \in \mathbb{R}^{M \times d}
$$

Then, the final question representation can be obtained by a weighted sum of these vectors.

$$
\begin{aligned}
w_{i j} & =\sigma\left(W_{q}^{T}\left[u_{i}^{l} ; p_{j}\right]+b_{q}\right) \\
\alpha_{i j} & =\frac{\exp \left(w_{i j}\right)}{\sum_{k=1}^{M} \exp \left(w_{i k}\right)} \\
q_{i}^{l} & =\sum_{j=1}^{M} \alpha_{i j} p_{j}
\end{aligned}
$$

Finally, $u_{i}^{l}$ could be updated via:

$$
\begin{aligned}
& \beta_{i}^{l}=\sigma\left(W_{s}^{T}\left[q_{i}^{l} ; u_{i}^{l}\right]+b_{s}\right) \\
& u_{i}^{l}=\beta_{i}^{l} \odot \tanh \left(q_{i}^{l}\right)+\left(1-\beta_{i}^{l}\right) \odot u_{i}^{l}
\end{aligned}
$$

With this new $u_{i}^{l}$, we use Equation 1 and 2 to obtain $h_{i}^{l+1}$, which is the hidden state of node $i$ at the $(l+1)$ th layer.

We stack the networks for $L$ layers where all parameters are shared, and finally obtain $h^{L}=\left\{h_{i}^{L}\right\}_{i=1}^{T}$ for the entity graph.

\subsection{Output Layer}

Similar to BAG [Cao et al., 2019], we apply a bi-directional attention between the entity graph and the question. The similarity matrix $S \in \mathbb{R}^{T \times M}$ is first calculated via

$$
S=a v g_{-1} f_{a}\left(\left[h^{L} ; p ; h^{L} \odot p\right]\right)
$$

where $a v g_{-1}$ is the average operation in last dimension, and $f_{a}$ is a single-layer MLP. Then, the node-to-question attention $g_{n 2 q}$ and question-to-node attention $g_{q 2 n}$ are calculated via

$$
\begin{aligned}
& g_{n 2 q}=\operatorname{softmax}_{c o l}(S) \cdot p \\
& g_{q 2 n}=\operatorname{dup}\left(\operatorname{softmax}\left(\max _{\mathrm{col}}(S)\right)\right)^{T} \cdot h^{L}
\end{aligned}
$$

where softmax $c$ col and $\max _{c o l}$ denote performing softmax and max function across columns respectively, dup is the function to duplicate the result $\operatorname{softmax}\left(\max _{c o l}(S)\right) \in$ $\mathbb{R}^{1 \times M}$ for $T$ times into shape $\mathbb{R}^{T \times M}$.

The output of the bi-directional attention layer is $\left[h^{L} ; g_{n 2 q} ; h^{L} \odot g_{n 2 q} ; h^{L} \odot g_{q 2 n}\right]$, which is then fed to a 2layer fully connected feed-forward network with tanh as the 
activation function in each layer. Finally, the softmax function is applied among the output, which can generate the prediction result for each node in the graph. Each candidate may correspond to several nodes, since it may appear in multiple documents. We use the maximal probability of these nodes as the result of the candidate, and use cross-entropy as the loss function.

\section{Experiments}

\subsection{Dataset and Experimental Settings}

We use WIKIHop [Welbl et al., 2018] to validate the effectiveness of our proposed approach, which is a multi-choice style reading comprehension data set. The dataset contain$\mathrm{s}$ about $43 \mathrm{~K} / 5 \mathrm{~K} / 2.5 \mathrm{~K}$ samples in training, development, and test set respectively. The test set is not public and can only be evaluated online blindly.

In our implementation, we use NLTK [Bird, 2006] to tokenize the supporting documents, question, and candidates into word sequences, and then find mentions of subject entity and candidates in supporting documents through the exact matching strategy. In order to extract reasoning entities, we use Stanford CoreNLP [Manning et al., 2014] to perform entity recognition on the supporting documents.

We use the standard 1024-dimension ELMo and 300dimension pre-trained GLoVe (trained from 840B Web crawl data) as word embeddings. The dimensions of hidden states in BiLSTM and GCN are set as $d=256$, and the number of nodes and the query length is truncated as 600 and 25 respectively. We stack $L=4$ layers of the Gated-RGCN blocks. During training, we set the mini-batch size as 16 , and use Adam [Kingma and Ba, 2015] with learning rate 0.0002 for learning the parameters.

\subsection{Main Results}

We compare our approach with several previously published models, and present our results in Table 2. The performance of multiple choice QA is evaluated by the accuracy of choosing the correct answer. Table 2 shows the performance of approaches both on development and test set respectively. As shown in the table, we can see that our approach achieves the state-of-the-art accuracy on both development and test set against all types of approaches, including GCN-based approaches (Entity-GCN [De Cao et al., 2019], BAG [Cao et al., 2019], HDE [Tu et al., 2019], MHQA-GRN [Song et al., 2018]), memory-based approaches (Coref-GRU [Dhingra et al., 2018], EPAr [Jiang et al., 2019]), path-based approaches (PathNet [Kundu et al., 2019]), and attention-based approaches (CFC [Zhong et al., 2019], DynSAN [Zhuang and Wang, 2019]).

For ensemble models, our approach also achieves state-ofthe-art performance, which surpasses the reported human performance [Welbl et al., 2018] by about $4.2 \%$.

\subsection{Ablations Studies}

We conduct an ablation study to evaluate the contribution of each model component, and show the results in Table 3.

In (a), we delete all reasoning entities, so our graph degenerates to the entity graph in [De Cao et al., 2019]. The

\begin{tabular}{lcc}
\hline Single Models & Dev & Test \\
\hline BIDAF [Seo et al., 2017] & 49.7 & 42.9 \\
Coref-GRU [Dhingra et al., 2018] & 56.0 & 59.3 \\
MHQA-GRN [Song et al., 2018] & 62.5 & 65.4 \\
Entity-GCN [De Cao et al., 2019] & 64.8 & 67.6 \\
PathNet ${ }^{\dagger}$ [Kundu et al., 2019] & 67.1 & - \\
BAG [Cao et al., 2019] & 66.5 & 69.0 \\
EPAr [Jiang et al., 2019] & 67.2 & 69.1 \\
CFC [Zhong et al., 2019] & 66.4 & 70.6 \\
HDE [Tu et al., 2019] & 68.1 & 70.9 \\
DynSAN [Zhuang and Wang, 2019] & 70.1 & 71.4 \\
\hline Proposed & $\mathbf{7 0 . 8}$ & $\mathbf{7 2 . 5}$ \\
\hline \hline Ensemble Models & & \\
\hline Entity-GCN [De Cao et al., 2019] & 68.5 & 71.2 \\
DynSAN ${ }^{\ddagger}$ [Zhuang and Wang, 2019] & - & 73.8 \\
HDE ${ }^{\ddagger}$ [Tu et al., 2019] & - & 74.3 \\
\hline Proposed & $\mathbf{7 4 . 0}$ & $\mathbf{7 8 . 3}$ \\
\hline Human & - & 74.1 \\
\hline
\end{tabular}

Table 2: The performance of different models on WIKIHOP dataset. "-" indicates missing results, $\dagger$ indicates that the missing results are not reported in their papers, $\ddagger$ indicates that the results are not reported in their papers but available on WIKIHOP leaderboard, and $\sharp$ indicates that the models are unpublished but only available on the leaderboard.

\begin{tabular}{lcc}
\hline Model & Dev & $\triangle$ \\
\hline Full model & $\mathbf{7 0 . 8}$ & - \\
\hline (a) w/o reasoning entities & 69.1 & -1.7 \\
(b) w/o question & 70.2 & -0.6 \\
(c) w/o attention in question encoding & 68.8 & -2.0 \\
(d) w/o edge types & 69.0 & -1.8 \\
(e) reduce edge types & 69.5 & -1.3 \\
\hline
\end{tabular}

Table 3: Ablation results on the WIKIHOP dev set.

accuracy on the development set drops to $69.1 \%$, but it is still higher than the accuracy of Entity-GCN [De Cao et al., 2019]. This proves the effectiveness of reasoning entities and the Gated-RGCN mechanism. In (b), we do not bring in the question in the reasoning. While in (c), we only use BiLSTM to encode the question, but do not adopt the attention mechanism in BiLSTM. From (b) and (c), we can see that question is useful for reasoning. In (d), we treat all edge types equally, so Gated-RGCN is replaced by Gated-GCN in reasoning, which reduces $1.8 \%$ accuracy absolutely. In (e), we only define the edge types as in BAG [Cao et al., 2019], and the accuracy drops to $69.5 \%$. From (d) and (e), we learn that different types of edges is also critical in the reasoning.

\subsection{Analysis}

In this section, we conduct a series of experiments with different setting in our approach. 


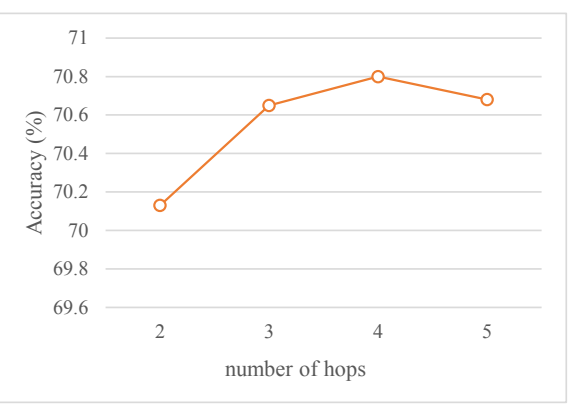

(a) number of hops

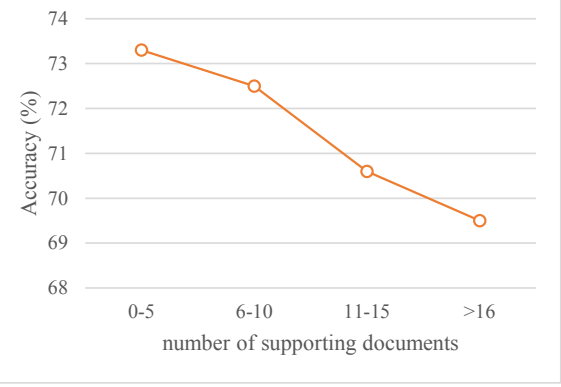

(b) number of supporting docs

Figure 3: The results of our approach on the WIKIHOP dev dataset with different experimental settings.

Different number of Gated-RGCN Layers. We evaluate our approach with different number of GCN layers $(L)$, and the result is shown in Figure 3(a). From the figure, we can see that the accuracy increases gradually and then drops finally, which proves the effectivity of Gated-RGCN. It is expected that the accuracy drops finally, because more hops would bring in noise. As for why 4-hops is the best, this may be because many samples are 1-hop problems, and the length of path in these samples is exactly 4.

Different number of supporting documents. We split the development set into subsets according to the number of supporting documents, and then evaluate our approach on each subset. The results are shown in Figure 3(b). From the figure, we can see that more documents may bring in more irrelevant information, which is harmful to the multi-hop QA. However, even on a number greater than 16, our model achieved an accuracy of $69.5 \%$, which is better than the overall effect of most models.

Different word embeddings. Table 4 shows the results of our approach when using different word embeddings. From the table, we can see that only using ELMo or GLoVe in our approach will cause a severe drop, and replacing ELMo with BERT [Devlin et al., 2019] delivers a competitive result. Meanwhile, we can see that for the GCN-based approach, the initialization of nodes is extremely critical, because this type of approach has greatly compressed the information when constructing the graph.

\begin{tabular}{lc}
\hline Embeddings & Dev \\
\hline ELMo & 66.1 \\
GLoVe & 64.5 \\
Bert+GLoVe & 70.78 \\
ELMo+GLoVe & $\mathbf{7 0 . 8 1}$ \\
\hline
\end{tabular}

Table 4: The results with different word embeddings on the WIKIHoP dev set.

\section{Conclusion}

In this paper, we propose a novel approach for multi-hop reading comprehension across documents. Our approach extends the entity graph by introducing reasoning entities, which can form the reasoning path from question to candidates. In addition, our approach incorporates the question in the multi-hop reasoning through a new gate mechanism to regulate how much useful information propagating from neighbors to the node. Experiments show that our approach achieves state-of-the-art accuracy both for single and ensemble models.

Our future work would focus on the interpretability of the multi-hop reading comprehension across documents. In addition, we would like to build the entity graph with reasoning entities dynamically during the reasoning, and apply our model on other datasets.

\section{Acknowledgments}

This work is supported by the National Key Research and Development Project of China (No. 2018AAA0101900), the Fundamental Research Funds for the Central Universities (No. 2019FZA5013), the Zhejiang Provincial Natural Science Foundation of China (No. LY17F020015), the Chinese Knowledge Center of Engineering Science and Technology (CKCEST) and MOE Engineering Research Center of Digital Library.

\section{References}

[Bird, 2006] Steven Bird. Nltk: the natural language toolkit. In Proceedings of the COLING/ACL on Interactive presentation sessions, pages 69-72. Association for Computational Linguistics, 2006.

[Cao et al., 2019] Yu Cao, Meng Fang, and Dacheng Tao. Bag: Bi-directional attention entity graph convolutional network for multi-hop reasoning question answering. In NAACL-HLT, 2019.

[Das et al., 2017] Rajarshi Das, Shehzaad Dhuliawala, Manzil Zaheer, Luke Vilnis, Ishan Durugkar, Akshay Krishnamurthy, Alexander J. Smola, and Andrew McCallum. Go for a walk and arrive at the answer: Reasoning over paths in knowledge bases using reinforcement learning. In ICLR, 2017.

[De Cao et al., 2019] Nicola De Cao, Wilker Aziz, and Ivan Titov. Question answering by reasoning across documents 
with graph convolutional networks. In Proceedings of the 2019 Conference of the North American Chapter of the Association for Computational Linguistics: Human Language Technologies, Volume 1 (Long and Short Papers), pages 2306-2317, 2019.

[Devlin et al., 2019] Jacob Devlin, Ming-Wei Chang, Kenton Lee, and Kristina Toutanova. Bert: Pre-training of deep bidirectional transformers for language understanding. In NAACL-HLT, 2019.

[Dhingra et al., 2018] Bhuwan Dhingra, Qiao Jin, Zhilin Yang, William W. Cohen, and Ruslan Salakhutdinov. Neural models for reasoning over multiple mentions using coreference. In NAACL-HLT, 2018.

[Hochreiter and Schmidhuber, 1997] Sepp Hochreiter and Jürgen Schmidhuber. Long short-term memory. Neural Computation, 9:1735-1780, 1997.

[Hu et al., 2017] Minghao Hu, Yuxing Peng, Zhen Huang, Xipeng Qiu, Furu Wei, and Ming Zhou. Reinforced mnemonic reader for machine reading comprehension. In IJCAI, 2017.

[Jiang et al., 2019] Yichen Jiang, N. Joshi, Yen-Chun Chen, and Mohit Bansal. Explore, propose, and assemble: An interpretable model for multi-hop reading comprehension. In $A C L, 2019$.

[Khashabi et al., 2018] Daniel Khashabi, Snigdha Chaturvedi, Michael Roth, Shyam Upadhyay, and Dan Roth. Looking beyond the surface: A challenge set for reading comprehension over multiple sentences. In NAACL-HLT, 2018.

[Kingma and Ba, 2015] Diederik P. Kingma and Jimmy Ba. Adam: A method for stochastic optimization. In ICLR, 2015.

[Kociský et al., 2017] Tomás Kociský, Jonathan Schwarz, Phil Blunsom, Chris Dyer, Karl Moritz Hermann, Gábor Melis, and Edward Grefenstette. The narrativeqa reading comprehension challenge. Transactions of the Association for Computational Linguistics, 6:317-328, 2017.

[Kundu et al., 2019] Souvik Kundu, Tushar Khot, Ashish Sabharwal, and Peter Clark. Exploiting explicit paths for multi-hop reading comprehension. In $A C L$ (1), pages 2737-2747. Association for Computational Linguistics, 2019.

[Lv et al., 2019] Xin Lv, Yuxian Gu, Xu Han, Lei Hou, Juanzi Li, and Zhiyuan Liu. Adapting meta knowledge graph information for multi-hop reasoning over few-shot relations. ArXiv, abs/1908.11513, 2019.

[Manning et al., 2014] Christopher D. Manning, Mihai Surdeanu, John Bauer, Jenny Rose Finkel, Steven Bethard, and David McClosky. The stanford corenlp natural language processing toolkit. In $A C L, 2014$.

[Mihaylov et al., 2018] Todor Mihaylov, Peter Clark, Tushar Khot, and Ashish Sabharwal. Can a suit of armor conduct electricity? a new dataset for open book question answering. In EMNLP, 2018.
[Pennington et al., 2014] Jeffrey Pennington, Richard Socher, and Christopher D. Manning. Glove: Global vectors for word representation. In EMNLP, 2014.

[Peters et al., 2018] Matthew E Peters, Mark Neumann, Mohit Iyyer, Matt Gardner, Christopher Clark, Kenton Lee, and Luke Zettlemoyer. Deep contextualized word representations. In Proceedings of NAACL-HLT, pages $2227-$ 2237, 2018.

[Qiu et al., 2019] Lin Qiu, Yunxuan Xiao, Yanru Qu, Hao Zhou, Lei Li, Weinan Zhang, and Yong Yu. Dynamically fused graph network for multi-hop reasoning. In $A C L$, 2019.

[Seo et al., 2017] Minjoon Seo, Aniruddha Kembhavi, Ali Farhadi, and Hannaneh Hajishirzi. Bidirectional attention flow for machine comprehension. In ICLR, 2017.

[Shen et al., 2017] Yelong Shen, Po-Sen Huang, Jianfeng Gao, and Weizhu Chen. Reasonet: Learning to stop reading in machine comprehension. In Proceedings of the $23 \mathrm{rd}$ ACM SIGKDD International Conference on Knowledge Discovery and Data Mining, pages 1047-1055. ACM, 2017.

[Song et al., 2018] Linfeng Song, Zhiguo Wang, Mo Yu, Yue Zhang, Radu Florian, and Daniel Gildea. Exploring graphstructured passage representation for multi-hop reading comprehension with graph neural networks. ArXiv, abs/1809.02040, 2018.

[Tu et al., 2019] Ming Tu, Guangtao Wang, Jing Huang, Yun Shu Tang, Xiaodong He, and Bowen Zhou. Multihop reading comprehension across multiple documents by reasoning over heterogeneous graphs. In $A C L, 2019$.

[Welbl et al., 2018] Johannes Welbl, Pontus Stenetorp, and Sebastian Riedel. Constructing datasets for multi-hop reading comprehension across documents. Transactions of the Association for Computational Linguistics, 6:287302, 2018.

[Williams, 1992] Ronald J. Williams. Simple statistical gradient-following algorithms for connectionist reinforcement learning. Machine Learning, 8:229-256, 1992.

[Yang et al., 2018] Zhilin Yang, Peng Qi, Saizheng Zhang, Yoshua Bengio, William W. Cohen, Ruslan Salakhutdinov, and Christopher D. Manning. Hotpotqa: A dataset for diverse, explainable multi-hop question answering. In EMNLP, 2018.

[Zhong et al., 2019] Victor Zhong, Caiming Xiong, Nitish Shirish Keskar, and Richard Socher. Coarse-grain fine-grain coattention network for multi-evidence question answering. In ICLR, 2019.

[Zhuang and Wang, 2019] Yimeng Zhuang and Huadong Wang. Token-level dynamic self-attention network for multi-passage reading comprehension. In ACL, 2019. 